\title{
Performance Comparison of Small Cell and Distributed Antenna Systems for In-Building Mobile Communications
}

\author{
Temitope Alade* and Qasim Zeeshan Ahmed ${ }^{\dagger}$ \\ * University of Worcester, ${ }^{\dagger}$ University of Huddersfield, United Kingdom
}

\begin{abstract}
To bring fifth generation (5G) mobile communication capabilities to indoor users, small cells and distributed antenna systems are two promising technologies being considered. This paper investigates and compares the performance of indoor deployed small cell base station (SBS) and indoor distributed antenna systems (DAS) in an isolated multi-storey building. Each floor of the building is equipped with either an indoor SBS or an indoor DAS where geographically distributed remote radio heads (RRHs) are connected to a central unit (CU), and frequency reuse is employed among floors. Signal propagation characteristics within multi-storey buildings and the impact of inter floor interference on performance is analysed and compared for both systems. The effect of different reuse distances, pathloss exponents, penetration loss and co-channel interference on achievable rate is analysed over a wide range of potential mobile equipment (ME) locations.
\end{abstract}

Index Terms-Small cells, distributed antenna system (DAS), do-channel interference, multi-floor in-building propagation, spectral efficiency.

\section{INTRODUCTION}

Fifth generation (5G) mobile communication systems promise high data rates, ultra reliable and low latency communication to all connected devices, but achieving these objectives remains a challenge particularly in regions with large number of users, such as urban/metropolitan areas and within buildings. Providing reliable system performance in these regions is difficult as, not only is the user density at its greatest, the communication traffic density and the capacity demand can be extremely high. Furthermore, because the base station transmitters are generally located outside of buildings, penetration losses are often encountered as the signal moves deeper into the interior of large buildings, thus limiting system performance and capacity [1].

To support increasing wireless traffic density within buildings, in-building wireless communication systems that will use small cells and employ extensive frequency reuse have been suggested. Among these are small cell base-stations (SBS) [2] and distributed antenna systems (DAS) [3] deployed within small buildings, or across several floors of multi-storey buildings [4].

Indoor SBS are typically low-powered, short-range (10$50 \mathrm{~m}$ ) cellular network base stations deployed within buildings, where each base station is a unique physical cell.
However, to support a large number of users, multiple SBS are often required [5], necessitating the reuse of frequency spectrum. Frequency reuse introduces cochannel interference, which severely limits the system performance, reducing the reliability, maximum transmission rates, and number of users that can be supported [6].

In indoor DAS, remote radio heads (RRHs) are deployed to supplement coverage and improve system performance across several floors within a building. In contrast to indoor SBS where all service antennas are located in a compact area, the RRH are geographically distributed. Indoor DAS effectively shortens the transmission distance between the transmitter and the receiver, and reduces path loss, transmit power, and cochannel interference [7] particularly for those mobile equipment (ME) near the edge of a cell [8].

For outdoor scenarios where cell sizes are generally within thousands of meters, [9] and [10] studied the performance of cellular networks with co-located and distributed base-station antennas. The studies indicated that the distributed antenna layout has potential for higher data rates than co-located antenna layout. However, results obtained from these studies cannot be applied directly to in-building scenarios where a large number of potential users are distributed throughout a relatively small three-dimensional (3D) space. Moreover, signal propagation in indoor environments occurs over shorter ranges than outdoor macro and microcellular systems and large losses can be experienced over very short distances. This is a major concern in multi-storey buildings where the same frequency channels may be reused on adjacent floors of the building to support a large number of users. Severe co-channel interference can occur and greatly limit the performance of the indoor system. Therefore, a thorough assessment of the way co-channel interference interacts with the $3 \mathrm{D}$ in-building environment is vital when planning indoor mobile communication systems.

This paper compares the downlink performance of indoor deployed SBS and indoor DAS employing frequency reuse in a multi-storey building. The systems are characterised under key factors including reuse distances, pathloss exponents and penetration loss. Analysis of both line-of-sight (LOS) and non-LOS (NLOS) propagation conditions is considered. To the authors' best knowledge, this network setup has not been analysed previously for an isolated multi-storey building. 


\section{RADIO PROPAGATION AND INTERFERENCE IN INDOOR ENVIRONMENTS}

Radio propagation in indoor environments differs from outdoor environments in a number of different ways [11]. For example, the distance between the transmitter and receiver, as well as propagation path lengths are typically much smaller (between $0-50 \mathrm{~m}$ ); indoor environments are inherently 3D in topology and arguably more cluttered; both LOS and NLOS propagation paths exist, consequently, large losses can be experienced over a short distance. Signal losses in indoor environments are determined by floor plans, construction materials used, peoples' positions and movements, type and number of office equipment, scale of MEs used, and so on. Propagation studies presented in [12] for multi-storey buildings indicate that interference can arise via three propagation paths: 1) internal paths that involve transmission through the floor partitions, which may include multiple reflections between the walls, floors and ceilings; 2) external paths that involve transmission via diffraction down the window edges; 3) external paths that involve signal reflections and scattering from a nearby building. In an isolated multi-storey building (Fig. 1), the diffraction down the window edges is the only external path possible. The impact of diffraction is insignificant and can be safely ignored [12].

\section{INDOOR SMALL-CELl SyStem Model}

Fig. 1(a) shows the downlink transmission of an indoor small-cell system in a multi-storey building where each floor of the building has one SBS centrally mounted on the ceiling to serve one ME on that floor. Each floor of the building has an open floor space plan and MEs are transmitting with the same frequency on different floors. The worst case downlink performance of the indoor small-cell system is likely to occur when a ME is located in the corner of a floor, because at this location, the desired signal power will be weakest.

In the building, it is assumed there are a total of $K$ SBS, one in each floor, $K$ also denotes the number of floors in the building and each SBS is equipped with $M$ co-located antennas. It is assumed that each ME has a single antenna and the desired ME is located on the 4th floor of the building. Each ME is located at a height of $v$ meters above each floor and an inter-floor spacing of $F$ meters is assumed. The reuse distance $C$ measured in floors, is the distance at which the frequency resource can be reused (Fig. 1 illustrates a reuse distance of three floors).

The transmitted signal from SBS- $k$ to the reference user is given by

$$
X_{k, m}(t)=\sqrt{P_{s}} \sum_{i=-\infty}^{\infty} b_{k, m}[i] \rho_{T_{s}}\left(t-i T_{s}\right)
$$

where $P_{s}$ denotes the transmit power which is assumed to be the same for all SBS, $b_{k, m}[i]$ is the transmitted symbol, $T_{s}$ represent the symbol duration, $E\left[b_{k, m}\right]=0$ and $E\left[b_{k, m}\right]^{2}=$ 1. $\rho_{T_{s}}(t)$ is a pulse waveform defined as $\rho_{T_{s}}(t)=1$ for $0 \leq t \leq T_{s}$ and $\rho_{T_{s}}(t)=0$ otherwise.

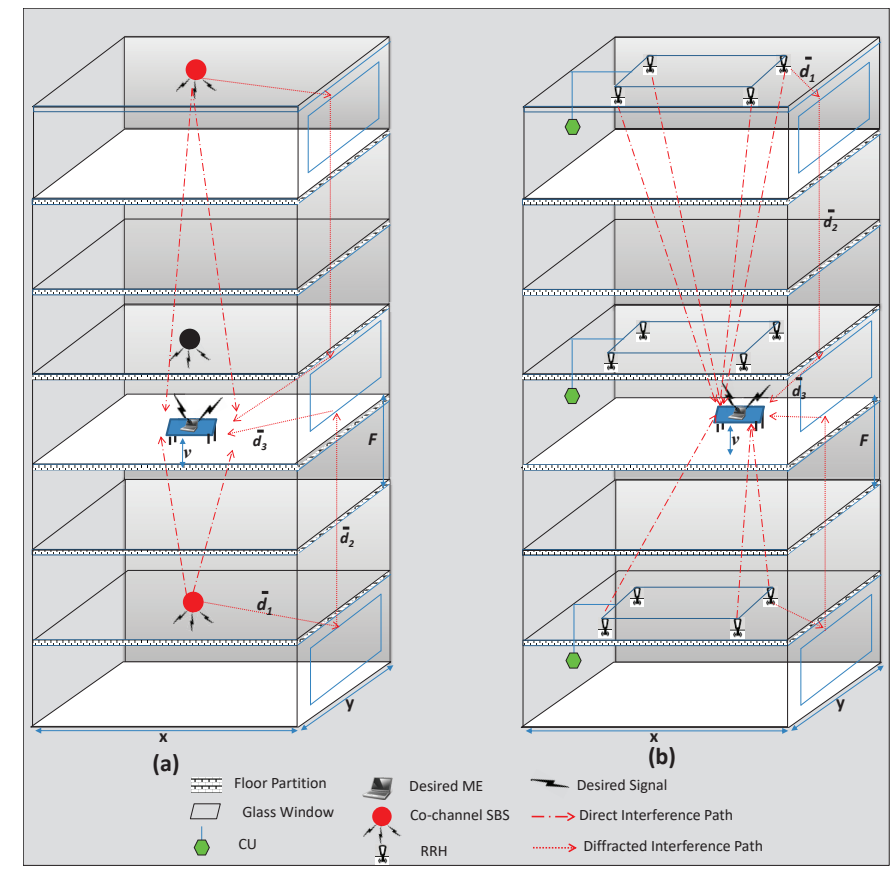

Fig. 1: (a) Indoor SBS with Frequency Reuse. (b) Indoor DAS with Frequency Reuse

The channel between the SBS- $k$ and reference user on the middle floor is given by the low pass equivalent impulse response as

$$
h_{k, m}(t)=\left(d_{k, m}\right)^{-\mu / 2} \cdot \tilde{\varphi}_{k, m}^{l / 2} \cdot \alpha_{k, m} \cdot e^{j \theta_{k, m}} \cdot \delta\left(t-\tau_{k, m}\right)
$$

where $d_{k, m}$ is the 3D path lengths from a SBS- $k$ to the desired ME. In (2), $\mu$ is the path loss exponent, typically varies between 2 to 6 depending on the physical layout and construction of the building [13]. $\tilde{\varphi}_{k, m}$ is the penetration loss through a single floor and $l$ is the number of floors in the transmission path. $\alpha_{k, m}, \theta_{k, m}$ and $\tau_{k, m}$ are the channel fading factor, path phase, and path delay, respectively, and are statistically independent. It is assumed that $\theta_{k, m}$ and $\tau_{k, m}$ are uniformly distributed over $[0,2 \pi]$ and $\left[0, T_{s}\right]$, respectively. $\delta(t)$ denotes the Dirac delta function. Assuming a Cartesian coordinate system, where $\left(x_{k}, y_{k}\right)$ represents the coordinate of the SBS- $k$, and $(x, y)$ denotes the coordinate of the the desired ME, $d_{k, m}$ can be written as

$$
d_{k, m}=\sqrt{\left(x_{k}-x\right)^{2}+\left(y_{k}-y\right)^{2}+[F l+(v-F)]^{2}}
$$

Accordingly, the received lowpass equivalent signal at the reference user is given by

$$
\begin{aligned}
y(t) & =\sum_{k=1}^{K} \sum_{m=1}^{M} X_{k, m}(t) \otimes h_{k, m}(t)+Z_{0}(t) \\
& =\sum_{k=1}^{K} \sum_{m=1}^{M} Q_{k, m} \cdot X_{k, m}\left(t-\tau_{k, m}\right)+Z_{0}(t)
\end{aligned}
$$

where the notation $\otimes$ denotes the convolution operation, $Q_{k, m}=\left(d_{k, m}\right)^{-\mu / 2} \cdot \tilde{\varphi}_{k, m}^{l / 2} \cdot \alpha_{k, m} \cdot e^{j \theta_{k, m}}$ and $Z_{0}(t)$ is the additive white Gaussian noise (AWGN) with zero mean and a double sided power spectral density $N_{0} / 2$. 
Assuming the phase and the path delay of the desired signal are precisely known at the receiver of the reference ME i.e, $\tau_{4, m}=0$, the demodulated signal over one symbol period $T_{s}$ is given by

$$
R=\frac{1}{T_{s}} \int_{0}^{T_{s}} y(t) \cdot e^{-j \theta_{k, m}} d t=S_{0}+I_{t}+Z
$$

where $S_{0}$ denotes the desired signal component received from the SBS on the reference floor, given by

$$
\begin{aligned}
S_{0}=\frac{1}{T_{s}} \int_{0}^{T_{s}}\left(d_{k^{\prime}, m}\right)^{-\mu / 2} \cdot \alpha_{k^{\prime}, m} \cdot e^{j \theta_{k^{\prime}, m}} \\
\cdot X_{k^{\prime}, m}(t) \cdot e^{-j \theta_{k^{\prime}, m}} d t \\
=\sqrt{P_{s}} \cdot \sum_{m=1}^{M}\left(d_{k^{\prime}, m}\right)^{-\mu / 2} \cdot b_{k^{\prime}, m} \cdot \alpha_{k^{\prime}, m}
\end{aligned}
$$

Note that the superscript ' refers to desired floor parameters. $I_{t}$ in (5) is the total co-channel interference term received by the reference user, given by

$$
I_{t}=\sum_{k=1, k \neq k^{\prime}}^{K} I_{k, m}
$$

where $I_{k, m}$ is the co-channel interference component from other floors, given by

$$
\begin{aligned}
I_{k, m} & =\frac{1}{T_{s}} \int_{0}^{T_{s}} Q_{r} \cdot X_{k, m}\left(t-\tau_{k, m}\right) \cdot e^{-j \theta_{k^{\prime}, m}} d t \\
& =\frac{Q_{k}}{T_{s}} \cdot \int_{0}^{T_{s}} X_{k, m}\left(t-\tau_{k, m}\right) d t
\end{aligned}
$$

where $Q_{r}=\left(d_{k, m}\right)^{-\mu / 2} \cdot \tilde{\varphi}_{k, m}^{l / 2} \cdot \alpha_{k, m} \cdot e^{j \theta_{k, m}}, Q_{k}=$ $\left(d_{k, m}\right)^{-\mu / 2} \cdot \tilde{\varphi}_{k, m}^{l / 2} \cdot \alpha_{k, m} \cdot e^{j\left(\theta_{k, m}-\theta_{k^{\prime}, m}\right)}$. Since $E\left[\alpha_{k, m}^{2}\right]=1$, $E\left[b_{k, m}\right]=0, E\left[b_{k, m} \cdot b_{k, m}^{*}\right]=1$ and $E\left[\tau_{k, m}^{2}\right]=E\left[\left(T_{s}-\right.\right.$ $\left.\left.\tau_{k, m}\right)^{2}\right]=\frac{T_{s}^{2}}{3}$, the real and imaginary parts of $I_{k, m}$ have the same variance derived as

$$
\sigma_{I_{k, m}}^{2}=\frac{P_{s}}{3} \cdot\left(d_{k, m}\right)^{-\mu} \cdot \tilde{\varphi}_{k, m}^{l}
$$

The total co-channel interference term $I_{t}$ is a zero mean Gaussian distributed random variable as shown in [14]and [15]. Thus, the expectation of $I_{k, m}$ is a sum of complex Gaussian random variables easily derived as $E\left[I_{t}\right]=0$ and the variance denoted by $\sigma_{I_{t}}^{2}$ is derived as

$$
\begin{aligned}
\sigma_{I_{t}}^{2} & =E\left[I_{t} \cdot I_{t}^{*}\right] \\
& =\frac{P_{s}}{3 \cdot M} \sum_{k=1, k \neq k^{\prime}}^{K} \sum_{m=1}^{M}\left(d_{k, m}\right)^{-\mu} \cdot \tilde{\varphi}_{k, m}^{l}
\end{aligned}
$$

$Z$ in (5) is the noise component with zero mean and a variance of $\sigma_{Z}^{2}=N_{0} /\left(4 T_{s}\right)$, which is assumed to be equal for all SBS. Thus, using (6) and (10), the instantaneous signal to interference plus noise ratio (SINR) $\gamma$ at the reference ME is given by

$$
\begin{aligned}
\gamma & =\frac{S_{0}^{2}}{\sigma_{I_{t}}^{2}+\frac{N_{0}}{4 T_{s}}} \\
& =\frac{P_{s} \cdot\left(d_{k^{\prime}, m}\right)^{-\mu} \cdot \alpha_{k^{\prime}, m}^{2}}{\frac{P_{s}}{3 M} \sum_{k=1, k \neq k^{\prime}}^{K} \sum_{m=1}^{M}\left(d_{k, m}\right)^{-\mu} \cdot \tilde{\varphi}_{k, m}^{l}+\frac{N_{0}}{4 T_{s}}}
\end{aligned}
$$

According to various propagation studies, the possibility of a LOS propagation is somewhat difficult to predict within buildings, particularly for SBS with colocated antennas [16]. Consequently, it is assumed that the small scale fading $\alpha_{k^{\prime}}$ is Rayleigh distributed. Thus, $\alpha_{k^{\prime}}^{2}$ is exponentially distributed and the pdf of $\gamma$ in (11) is given by

$$
P_{\gamma}(\gamma)=\frac{1}{\bar{\gamma}} \exp \left(-\frac{\gamma}{\bar{\gamma}}\right)
$$

where $\bar{\gamma}$ is the average SINR for the reference ME given by

$$
\bar{\gamma}=\frac{\frac{4 E_{s}}{N_{0}}}{\frac{4}{3 M} \cdot \frac{E_{s}}{N_{0}} \sum_{k=1, k \neq k^{\prime}}^{K} \sum_{m=1}^{M}\left(\frac{d_{k, m}}{d_{k^{\prime}, m}}\right)^{-\mu}+1}
$$

$\frac{E_{s}}{N_{0}}$ in (13) is the average received symbol energy-to-noise density ratio at the $\mathrm{ME}$ receiver location and $E_{s}$ is expressed as

$$
E_{s}=P_{s} \cdot T_{s} \cdot d_{k^{\prime}, m}^{-\mu}
$$

Therefore, the maximum possible rate of the indoor SBS system per floor for a giving location of the reference ME is given by

$$
C_{T}=\int_{0}^{\infty} C_{b} \cdot \log _{2}(1+\gamma) P_{\gamma}(\gamma) d \gamma
$$

where $C_{b}=1 / T_{s}$ is the channel bandwidth. The conditional rate per $\mathrm{Hz}$ is given by

$$
C_{c}=\frac{C_{T}}{C_{b}}=\int_{0}^{\infty} \log _{2}(1+\gamma) P_{\gamma}(\gamma) d \gamma
$$

By substituting (12) into (16), the conditional achievable rate per $\mathrm{Hz} C_{c}$ is rewritten as

$$
\begin{aligned}
C_{c}=-\log _{2}(1+\gamma) \cdot \exp \left(-\frac{\gamma}{\bar{\gamma}}\right)^{\infty} \\
\quad+\int_{0}^{\infty} \frac{1}{(1+\gamma) \cdot \ln 2} \cdot \exp \left(-\frac{\gamma}{\bar{\gamma}}\right) d \gamma
\end{aligned}
$$

\section{INDOOR DAS MODEL}

In this section, the system model and achievable rate expressions for indoor DAS is presented.

For indoor DAS, the downlink transmission of the hypothetical building shown in Fig. 1(b) is considered, where each floor of the building is equipped with a CU comprised of $N$ evenly spaced, ceiling mounted RRHs to serve MEs on each floor. In order to make a fair comparison between the indoor SBS and the indoor DAS, the same building geometry, same number of antennas per floor, same total transmit power per floor and same total system bandwidth are assumed for both systems.

In the downlink, the reference ME located in the middle floor of the building is served by one or more RRH in order to improve the received signal strength via spatial diversity [17]. Due to frequency reuse, the performance of the indoor DAS will depend on the strength of the desired signal and 
the relative strength of the interfering signals originating from nearby floors.

In contrast to the indoor SBS, the signals transmitted from different RRHs experience different large scale fading. Consequently, an efficient power allocation over the RRHs is necessary in order to significantly enhance the SINR at the $\mathrm{ME}$ and increase the achievable rate. Several power allocation strategies have been proposed in literature including the well known water filling solution, an optimal power allocation that maximizes the capacity when the channel state information is available at the transmitter. However, the water filling solution is very complicated, thus a simplified scheme proposed in [18] is employed to allocate tranmit power to the RRHs. Accordingly, the transmit power to be allocated to the $n$th $\mathrm{RRH}$ on the reference floor is given by

$$
P_{n}=\frac{P_{s} \cdot\left(d_{k^{\prime}, n}\right)^{-\mu} \cdot \alpha_{k^{\prime}, n}^{2}}{\sum_{i=1}^{N}\left(d_{k^{\prime}, i}\right)^{-\mu} \cdot \alpha_{k^{\prime}, i}^{2}}
$$

where $d_{k^{\prime}, n}$ denotes the distance between the $n$th RRH and the ME on the reference floor. $d_{k, n}$ can be obtained following (3). Note that the number of intervening floors $l$ between the desired ME and its target RRH on the reference floor is zero (i.e., $l=0$ ). Assuming that the channel phase $\theta_{n}$ and the path delay $\tau_{n^{\prime}}$ are precisely known at the $n$th RRH on the reference floor, the transmitted signal can be expressed as

$X_{k, n}(t)=\sqrt{P_{n}} \cdot e^{-j \theta_{k, n}} \cdot \sum_{i=-\infty}^{\infty} b_{k, n}[i] \rho_{T_{s}}\left(t-i T_{s}+\tau_{k^{\prime}, n}\right)$

where $b_{k, n}$ denotes the transmitted symbol with $E\left[b_{k, n}\right]=0$ and $E\left[b_{k, n}\right]^{2}=1$, and assumed to be the same for all RRHs.

The channel between the $n$th $\mathrm{RRH}$ and the reference ME on the middle floor is modelled as a low pass equivalent impulse response, given by

$$
h_{k, n}(t)=\left(d_{k, n}\right)^{-\mu / 2} \cdot \tilde{\varphi}_{k, n}^{l / 2} \cdot \alpha_{k, n} \cdot e^{j \theta_{k, n}} \cdot \delta\left(t-\tau_{k, n}\right)
$$

where $\theta_{k, n}$ and $\tau_{k, n}$ are the path phase and path delay between the $n$th RRH and the reference user respectively, and are statistically independent. Given the geographical distribution of the RRHs, it is assumed that there is a possibility of LOS propagation between the $n$th RRH and the ME on the reference floor. Thus $\alpha_{k, n}$ undergoes Nakagami fading, hence, $\alpha_{k, n}^{2}$ is Gamma distributed with the probability distribution function (pdf) expressed as [19].

$$
\begin{gathered}
p_{\alpha_{k, n}^{2}}\left(\alpha_{k, n}^{2}\right)=\left(\frac{m_{n}}{\Omega_{n}}\right)^{m_{n}} \frac{\left(\alpha_{k, n}^{2}\right)^{m_{n}-1}}{\Gamma\left(m_{n}\right)} \exp \left(-\frac{m_{n}}{\Omega_{n}} \alpha_{k, n}^{2}\right), \\
\alpha_{k^{\prime}, n} \geq 0
\end{gathered}
$$

where $m_{n}$ is the Nakagami factor and $\Omega_{n}$ is the average fading power of the received signal. The parameters $\Omega_{n}$ and $m_{n}$ can be expressed as $\Omega_{n}=E\left[\alpha_{k, n}^{2}\right]$ and $m_{n}=$ $\frac{\Omega_{n}^{2}}{E\left[\left(\alpha_{k, n}^{2}-\Omega_{n}\right)^{2}\right]}, m_{n} \geq \frac{1}{2}$ respectively. When $m_{n}=1, \mathrm{Na}-$ gakami fading reduces to Rayleigh fading [19].
Accordingly, the received lowpass equivalent signal at the reference $\mathrm{ME}$ is given by

$$
\bar{y}(t)=\sum_{k=1}^{K} \sum_{n=1}^{N} X_{k, n}(t) \otimes h_{k, n}(t)+\bar{Z}_{0}(t)
$$

Assuming the receiver has a perfect timing synchronisation with the RRHs on the reference floor, i.e $\tau_{k^{\prime}, n}=0$, the demodulated signal over one symbol period $T_{s}$ is given by

$$
\bar{R}=\frac{1}{T_{s}} \int_{0}^{T_{s}} \bar{y}(t) d t=\bar{S}_{0}+\bar{I}_{t}+\bar{Z}
$$

where $\bar{S}_{0}$ denotes the desired signal component received from all RRHs located on the reference floor, given by

$$
\begin{aligned}
\bar{S}_{0} & =\sqrt{\frac{P_{s}}{\sum_{n=1}^{N}\left[d_{n^{\prime}, i}\right]^{-\mu} \cdot \alpha_{n^{\prime}, i}^{2}}} \cdot b_{n^{\prime}, k} \cdot \sum_{n=1}^{\bar{N}}\left[d_{n^{\prime}, k}\right]^{-\mu} \cdot \alpha_{n^{\prime}, k}^{2} \\
& =\sqrt{P_{s}} \cdot b_{n^{\prime}, k} \cdot \sqrt{\sum_{n=1}^{N}\left[d_{n^{\prime}, k}\right]^{-\mu}} \cdot \alpha_{n^{\prime}, k}^{2}
\end{aligned}
$$

$\bar{I}_{t}$ is the total co-channel interference term received by the reference $\mathrm{ME}$, given by

$$
\bar{I}_{t}=\sum_{k=1, k \neq k^{\prime}}^{K} \sum_{n=1}^{N} \bar{I}_{k, n}
$$

where $\bar{I}_{k, n}$ is the co-channel interference component from other floors, given by

$$
\begin{aligned}
\bar{I}_{k, n} & =\frac{1}{T_{s}} \int_{0}^{T_{s}} Q_{d} \cdot X_{k, n}\left(t-\tau_{k, n}\right) d t \\
& =\frac{\bar{Q}_{d}}{T_{s}} \cdot \int_{0}^{T_{s}} X_{k, n}\left(t-\tau_{k, n}\right) d t
\end{aligned}
$$

where $Q_{d}=\left(d_{k, n}\right)^{-\mu / 2} \cdot \tilde{\varphi}_{k, n}^{l / 2} \cdot \alpha_{k, n} \cdot e^{j \theta_{k, n}}, \bar{Q}_{d}=\left(d_{k, n}\right)^{-\mu / 2}$. $\tilde{\varphi}_{k, n}^{l / 2} \cdot \alpha_{k, n} \cdot e^{j\left(\theta_{k, n}-\theta_{n^{\prime}, k}\right)}$. Similar to the analysis in Section III, since $E\left[\alpha_{k, n}^{2}\right]=1, E\left[b_{k, n}\right]=0, E\left[b_{k, n} \cdot b_{k, n}^{*}\right]=1$ and $E\left[\tau_{k, n}^{2}\right]=E\left[\left(T_{s}-\tau_{k, n}\right)^{2}\right]=\frac{T_{s}^{2}}{3}$, the real and imaginary parts of $\bar{I}_{k, n}$ have the same variance derived as

$$
\sigma_{\bar{I}_{k, n}}^{2}=\frac{P_{s}}{3 \cdot N} \cdot\left(d_{k, n}\right)^{-\mu} \cdot \tilde{\varphi}_{k, n}^{l}
$$

The total co-channel interference term $\bar{I}_{t}$ is a zero mean Gaussian distributed random variable [?] and the variance denoted by $\sigma_{\bar{I}_{t}}^{2}$ is derived as

$$
\begin{aligned}
\sigma_{\bar{I}_{t}}^{2} & =E\left[\bar{I}_{t} \cdot \bar{I}_{t}^{*}\right] \\
& =\frac{P_{s}}{3 \cdot N} \sum_{k=1, k \neq k^{\prime}}^{K} \sum_{n=1}^{N}\left(d_{k, n}\right)^{-\mu} \cdot \tilde{\varphi}_{k, n}^{l}
\end{aligned}
$$

$\bar{Z}$ in (24) is the noise component with a variance of $\sigma_{Z}^{2}=$ $N_{0} /\left(4 T_{s}\right)$, which is assumed to be equal for all RRHs.

The instantaneous SINR $\overline{\bar{\gamma}}$ at the reference ME is given by

$$
\overline{\bar{\gamma}}=\frac{{\overline{S_{0}}}^{2}}{\sigma_{\bar{I}_{t}}^{2}+\frac{N_{0}}{4 T_{s}}}=\sum_{n=1}^{N} \overline{\bar{\gamma}}_{n}
$$


where $\overline{\bar{\gamma}}_{n}$ is given by

$$
\overline{\bar{\gamma}}_{n}=\frac{\frac{4 \bar{E}_{s}}{N_{0}}}{\frac{4}{3 N} \cdot \frac{\bar{E}_{s}}{N_{0}} \sum_{k=1, k \neq k^{\prime}}^{K} \sum_{n=1}^{N}\left(\frac{d_{k, n}}{d_{k^{\prime}, n}}\right)^{-\mu} \cdot \tilde{\varphi}_{k, n}^{l}+1} \cdot \alpha_{n^{\prime}, k}^{2}
$$

$\frac{\bar{E}_{s}}{N_{0}}$ is the average received symbol energy-to-noise density ratio at the ME receiver location and $\bar{E}_{s}$ is expressed as

$$
\bar{E}_{s}=P_{s} \cdot T_{s} \cdot d_{k^{\prime}, n}^{-\mu}
$$

Assuming arbitrary values of the Nakagami fading parameters, the pdf of the instantaneous SINR, $\overline{\bar{\gamma}}_{n}$ in (32), is then obtained as [20]

$$
P \overline{\bar{\gamma}}(\overline{\bar{\gamma}})=\frac{1}{\pi} \int_{0}^{\infty} \frac{\cos \left[\sum_{n=1}^{N} m_{n} \tan ^{-1}\left(\frac{t}{\beta_{n}}\right)-t \overline{\bar{\gamma}}\right]}{\prod_{n=1}^{N}\left(1+\left(\frac{t}{\beta_{n}}\right)^{2}\right)^{m_{n} / 2}} d t
$$

where $\beta_{n}=m_{n} / \hat{\bar{\gamma}}_{n} \cdot \hat{\bar{\gamma}}_{n}$ is the average SINR per RRH given by

$$
\hat{\bar{\gamma}}_{n}=\frac{\frac{4 \bar{E}_{s}}{N_{0}}}{\frac{4}{3} \cdot \frac{\bar{E}_{s}}{N_{0}} \sum_{k=1, k \neq k^{\prime}}^{K} \sum_{n=1}^{N}\left(\frac{d_{k, n}}{d_{k^{\prime}, n}}\right)^{-\mu} \cdot \tilde{\varphi}_{k, n}^{l}+1} \cdot \Omega_{n}
$$

The maximum possible rate of the indoor DAS per floor for a giving location of the reference $\mathrm{ME}$ is given by

$$
\bar{C}_{T}=\int_{0}^{\infty} C_{b} \cdot \log _{2}(1+\bar{\gamma}) P_{\bar{\gamma}}(\overline{\bar{\gamma}}) d \bar{\gamma}
$$

The conditional rate per $\mathrm{Hz}$ is derived by substituting (32) into (34), written as

$$
\begin{aligned}
& \bar{C}_{c}=\int_{0}^{\infty} \log _{2}(1+\bar{\gamma}) \\
& \cdot \frac{1}{\pi} \int_{0}^{\infty} \frac{\cos \left[\sum_{n=1}^{N} m_{n} \tan ^{-1}\left(\frac{t}{\beta_{n}}\right)-t \overline{\bar{\gamma}}\right]}{\prod_{n=1}^{N}\left(1+\left(\frac{t}{\beta_{n}}\right)^{2}\right)^{m_{n} / 2}} d t
\end{aligned}
$$

\section{NUMERICAL RESULTS}

In this section, the achievable rate performance of the indoor SBS is compared with the indoor DAS using the analytic formulas derived in Sections III and IV for the hypothetical multi-storey building shown in Fig.1. For fair comparison, the same number of antennas per floor is assumed for both systems. MEs are assumed to be evenly located across the floor and 10000 possible ME locations are considered and used to numerically calculate the average value of the achievable rate across the entire floor. Unless otherwise stated, Table 1 presents the summary of parameters used in evaluating the performance of the systems.

Fig. 2 shows the average achievable rate of the indoor $\mathrm{SBS}$ and the indoor DAS for frequency reuse distances of
$C=1,2$ floors. Note that a frequency reuse distance of one floor implies that the same frequencies are reused every floor, while in a reuse distance of two floors, channels are reused every second floor (co-channel interfering users are located on the 3rd, 5th and 7th floors). Clearly, superior performance is obtained with the indoor DAS over the indoor SBS. This is due to lower transmission path loss to the desired ME. In the indoor DAS, interfering signals experience a higher distance dependent rate of attenuation than that observed in the indoor SBS. Consequently, the received power by the desired ME dominates the performance and the resulting achievable rate is higher than that achieved with indoor SBS. It can be seen that increasing the reuse distance from one floor to two floors improves the performance of both systems significantly, especially at high SNR regions. This is due to high inter-floor isolation of co-channel floors which ensures high SINR, and high achievable rate across the floor. At high SNR values, the indoor DAS provides nearly $1 \mathrm{Bits} / \mathrm{Sec} / \mathrm{Hz}$ increase in achievable rate over the indoor SBS for a reuse distance of 1 floor. Much larger achievable rates are observed for a reuse distance of two floors. At lower SNR values below $35 \mathrm{~dB}$, the achievable rate of both systems increase linearly and the the curves tend to be flat at SNR values more than about $40 \mathrm{~dB}$ because co-channel interference dominates channel noise. Note that the performance of the indoor SBS tends to flatten more rapidly at higher SNR values because the system becomes more interference limited.

Fig. 3 shows the average achievable rate of the indoor SBS and the indoor DAS for a range of floor penetration losses with respect to frequency reuse distances of $C=1,2$ floors. A significant reduction in the total received signal to interference power is due to floor penetration losses. The penetration loss introduced by each floor depends on thickness of the floor inside the building and can vary from building to building. It is observed from the figure that the achievable rate of the indoor DAS are consistently higher than those of the indoor SBS. For both systems, the achievable rate increases as the penetration loss values increases. This is due to increase in the attenuation of the interfering signals. Results indicates that the indoor SBS may not tolerate a reuse distance of one floor due to low inter-floor isolation which results in low SINR, and low achievable rate values. Therefore an indoor DAS is mandatory in such buildings to ensure reliable wireless service.

TABLE I: Summary of Parameters

\begin{tabular}{l|c}
\hline Parameters & Value \\
\hline \hline Number of floors in the building, $K$ & 7 \\
\hline Inter-floor spacing, $F$ & $4 \mathrm{~m}$ \\
\hline Floor dimension, $(x, y)$ & $40 \mathrm{~m} \mathrm{X} 40 \mathrm{~m}$ \\
\hline ME located across the floor at height, $v$ & $1 \mathrm{~m}$ \\
\hline Reuse distance, $C$ & 1 floor \\
\hline Number of RRH on each floor, $N$ & 4 \\
\hline Number of antenna on each floor, $M$ & 4 \\
\hline Path loss exponent, $\lambda$ & 2.5 \\
\hline Penetration loss, $\varphi$ & $13 \mathrm{~dB}$ \\
\hline Nakagami fading value, $m$ & $1.8,1.5,1.25,1.0$ \\
\hline Transmit SNR, $E_{s} / N_{0}$ & $30 \mathrm{~dB}$ \\
\hline
\end{tabular}




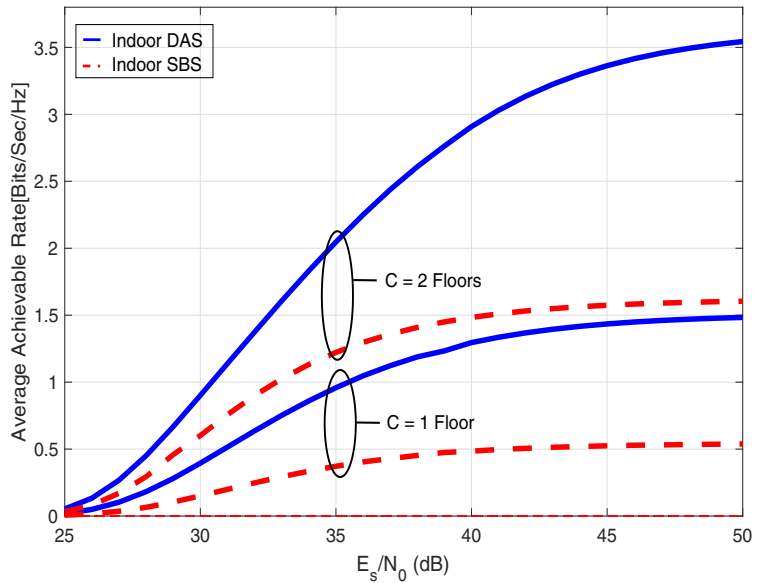

Fig. 2: Achievable rate comparison of indoor SBS and indoor DAS for different frequency reuse distances

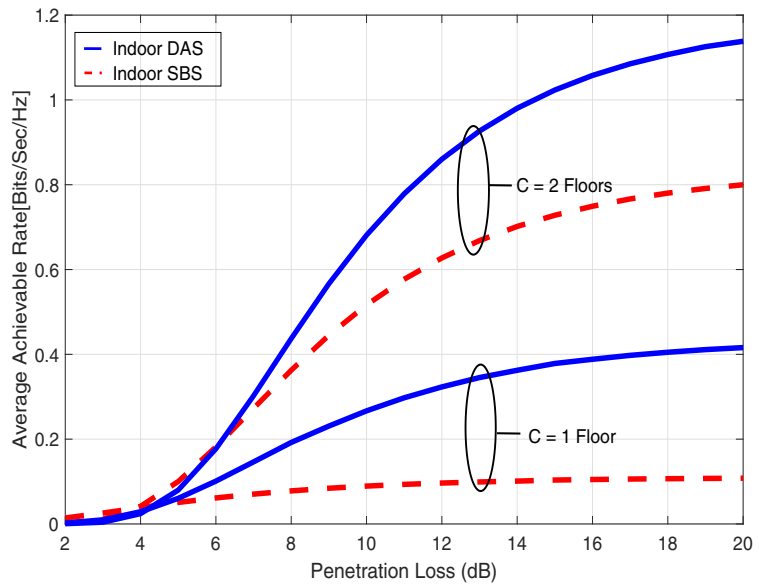

Fig. 3: Effect of penetration loss on achievable rate of indoor SBS versus indoor DAS for different reuse distances

\section{CONCLUSION}

Indoor mobile communication systems are required to provide high data rate transmissions for mobile users located inside the building. However, these indoor system are likely to be deployed in dense in-building environments where the frequency channels are reused. This paper has comparatively analysed the performance of indoor SBS and indoor DAS employing frequency reuse. The in-building environment is mathematically defined and the performance of both systems is analytically quantified in terms of achievable rate for a 7 storey building. The values of propagation parameters used are from published propagation studies. It is been identified that superior performance is obtained with the indoor DAS over the indoor SBS. It is shown that with indoor SBS in some buildings, it may be difficult to achieve adequate isolation between co-channel floors. For such buildings, an indoor DAS is therefore mandatory.

\section{REFERENCES}

[1] T. S. Rappaport, Y. Xing, G. R. MacCartney, A. F. Molisch, E. Mellios, and J. Zhang, "Overview of Millimeter Wave Communications for Fifth-Generation (5G) Wireless NetworksWith a Focus on Propagation Models", IEEE Transactions on Antennas and Propagation, vol. 65, pp. 62136230, December 2017.

[2] D. Lopez-Perez, M. Ding, H. Claussen, A. H. Jafari, "Towards 1 Gbps/UE in Cellular Systems: Understanding Ultra-Dense Small Cell Deployments", IEEE Communications Surveys \& Tutorials, vol. 17, no. 4, pp. 2078 - 2101, June 2015.

[3] Y. D. Beyene, R. Jantti, K. Ruttik, "Cloud-RAN Architecture for Indoor DAS", IEEE Access, vol. 2, pp. 1205 - 1212, Oct. 2014.

[4] T. Alade, H. Zhu, and J. Wang, "Uplink spectral efficiency analysis of in-building distributed antenna systems," IEEE Transactions on Wireless Communications, vol. 14, no. 7, pp. 4063-4074, July 2015.

[5] J. G. Andrews, H. Claussen, M. Dohler, S. Rangan, and M. C. Reed, "Femtocells: Past, present, and future", IEEE Journal on Selected Areas in Communications, vol. 30, no. 3, pp. 497 - 508, Apr. 2012.

[6] C. Zhan, and G. Yao, "Cooperative transmission in small cell networks using network coding", IEEE Wireless Communication Letters, vol. 7, no. 4, pp. 510 - 513, Aug. 2018.

[7] W. Choi and J. G. Andrews, "Downlink performance and capacity of distributed antenna systems in a multicell environment", IEEE Transactions on Wireless Communications, vol. 6, no. 1, pp. $69-73$, Jan. 2007.

[8] R. Heath, S. Peters, Y. Wang, and J. Zhang, "A current perspective on distributed antenna systems for the downlink of cellular systems", IEEE Communication Magazine vol. 51, no. 4, pp. 161 - 167, Apr. 2013.

[9] G. N. Kamga, M. Xia, and S. Aissa, "Spectral-efficiency analysis of massive mimo systems in centralized and distributed schemes," IEEE Transactions on Communications., vol. 64, no. 5, pp.1930-1941, May 2016.

[10] J. Li, D. Yue, and Y. Sun, "Performance analysis of millimeter wave massive mimo systems in centralized and distributed schemes," IEEE Access., vol. 6, pp.75482-75494, Nov. 2018.

[11] Recom. ITU-R P.1238-6,"Propagation data and prediction methods for the planning of indoor radiocommunication systems and radio local area networks in the frequency range $900 \mathrm{MHz}$ to $100 \mathrm{GHz}$ "; 2017.

[12] M. A. Panjwani, A. L. Abbott, and T. S. Rappaport, "Interactive computation of coverage regions for wireless communication in multifloored indoor environments," IEEE J. Select. Areas Commun., vol. 14, pp.420430, Apr. 1996.

[13] K. Haneda et al., Indoor 5G 3GPP-like channel models for office and shopping mall environments, in 2016 IEEE International Conference on Communications Workshops (ICC), May 2016, pp. 694699.

[14] Q. Z. Ahmed, K. Park, M. Alouini and S. Aissa, "Linear transceiver design for nonorthogonal amplify and forward protocol using a bit error rate criterion"; IEEE Transactions on Wireless Communications, vol. 13 , no. 4, pp. 1844-1853, Apr. 2014.

[15] Q. Z. Ahmed, K. Park, and M. Alouini, "Ultrawide bandwidth receiver based on a multivariate generalized gaussian distribution"; IEEE Transactions on Wireless Communications, vol. 14, no. 4, pp. 1800-1810, Apr. 2015

[16] C. Oestges, N. Czink, B. Bandemer, P. Castiglione, F. Kaltenberger, and A. J. Paulraj, "Experimental Characterization and Modeling of Outdoor-to-Indoor and Indoor-to-Indoor Distributed Channels", IEEE Transactions on Vehicular Technology, vol. 59, pp. 22532265, June 2010.

[17] J. Park, E. Song, and W. Sungs, "Capacity analysis for distributed antenna systems using cooperative transmission schemes in fading channels"; emphIEEE Trans. Wireless Commun., vol. 8, no. 2, pp. 586592, Feb. 2009

[18] Huiling Zhu, "Performance Comparison between Distributed Antenna and Microcellular Systems," IEEE Journal on Selected Areas in Communications, vol. 29, no. 6, pp. 1151-1163, June 2011.

[19] L. L. Yang and L. Hanzo, "Performance of generalized multicarrier DSCDMA over Nakagami-m fading channels," IEEE Trans. Commun. vol. 50, no. 6, pp. 956-966, Jun. 2002.

[20] G. Efthymoglou and V. Aalo, "Performance of RAKE receivers in Nakagami fading channel with arbitrary fading parameters", IEEE Electronic Letters, Vol.31, No.18, pp. 1610-1612, Aug. 1995. 